ACTA UNIVERSITATIS CIBINIENSIS - TECHNICAL SERIES

Vol. LXVII 2015

\title{
EXPERIMENTAL RESEARCH REGARDING THE MOTION CAPACITY OF A ROBOTIC ARM
}

\author{
DUMITRU Violeta Cristina \\ Assistant Professor/Ph.D., University of Craiova, Faculty of Mechanics, \\ Applied Mechanics and Civil Engineering Dept., dumitru.violeta@yahoo.com, Dolj, Romania
}

\begin{abstract}
This paper refers to the development of necessary experiments which obtained dynamic parameters (force, displacement) for a modular mechanism with multiple vertebrae. This mechanism performs functions of inspection and intervention in small spaces. Mechanical structure allows functional parameters to achieve precise movements to an imposed target. Will be analyzed the dynamic of the mechanisms using simulation instruments DimamicaRobot.tst under TestPoint programming environment and the elasticity of the tension cables. It will be changes on the mechanism so that spatial movement of the robotic arm is optimal.
\end{abstract}

Keywords: modular mechanism, design, testing, dynamic behavior, performance

\section{Introduction}

The robots play an important role in handling the tools. The integration of robotic systems in industrial fabrication and medicine, implicitly in restricted spaces, has been the center of the research for nearly two decades [1], [2]. Most popular research in robotics have been developed in modular robotics laboratories Tran M-II (Distributed Systems Design Research Group - Tsukuba, Japan), Hirose Titeche (Tokyo Institute of Technology), CONRO (University of Southern California - Los Angeles), Faculty of Automatic Control and Computers (University of Craiova).

Dario [3] presented an SMA with a degree of freedom that sag as snake for arthroscopy of the knee. Recently, Reynarts [4] presented a tool with two degrees of freedom and $5 \mathrm{~mm}$ in diameter, actuated by wires, using a tube of super elastic nitinol NiTi with flexing joints. Guthart and Salisbury [5] have developed a snake-like robot with a separate backbone and actuated by wire.

In robotic systems that perform the inspection and intervention in confined spaces, besides drive and control systems, appears a robotic arm with multiple joints, which actually performs the movement of an object in 3D space (tool, instrument).

Therefore, knowledge of the dynamic behavior of the robot arm, as part of the execution movement, shows interest in ensuring their performance. In this regard, besides theoretical research of dynamic behavior, an important role is the experimental research, designed to confirm, based on experimental measurements, the actual performance of the dynamic behavior of robots.

To experimental test of the robotic arm, in order to investigate the factors influencing the dynamic behavior of these elements, has been designed and developed a modular test stand. It was used modular components, to which were added other equipment, components and devices specially purchased to minimize the costs.

\section{Assumptions}

In general, the issue of the dynamic behavior of a mechanical system is reduced to analyze the movement. The transmission of the motion actuators to the end effector (distal vertebra) is facilitated by the drive system (linear slides, tension cables). The action forces translate into dynamic effects felt on the robotic arm. From qualitatively point of view, the degree of perception of the dynamic effects depends on the structural and functional configuration of the system.

Initial working hypothesis for the dynamic analysis that will be further developed are:

- system configuration is known;

- technical components have a well defined contribution; 
Thus, based on the initial hypothesis, it was simulated and analyzed, in detail, the internal mechanisms that determine and support the dynamics of the system under the action of external loads.

From the understanding of how the dynamic behavior is at the mechanical system (through its components), may result viable conclusions with direct effects on structural and technological optimization.

Experimental research approach consisted in planning experiments on spatial ability robotic arm motion as a function of forces and elasticity which manifests itself in tension cables of the mechanism using two types of disturbing signals, impulse and step. Research methods used were data acquisition and graphics.

\section{Experimental equipment}

For experiments, was designed and developed a modular test stand [6] [7]. In principle, this stand is composed of five major sub-assemblies, namely: poly-articulated robotic arm, drive system, management system, sensory system (transducers, sensors) and data acquisition system.

Each subsystem has been designed for easy integration and to facilitate the requirements. Also, each of the drive mechanism is driven continuously by a motor with torque $1.25 \mathrm{Nm}$ and speed $120 \mathrm{RPM}$, allowing a speed of advance / retreat of the robotic arm of $50 \mathrm{~mm} / \mathrm{s}$. Linear sleds are driven by two mechanisms (diameter $12 \mathrm{~mm}$, step of $3 \mathrm{~mm}$ ), which allow a linear displacement of the mechanism of \pm $172 \mathrm{~mm}$ in both directions. Stroke limiters are used for determining the position of the robotic arm. The operator operates the robotic arm control elements.

The considered robotic arm with ball joint, have a lot of adjacent vertebrae elements, interconnected by cables (wires) arranged in series. The vertebrae are held in contact by cables (wires) arranged at $120^{\circ}$ to each other. By operating the two wires of the mechanism is possible to rotate the final vertebra with an angle of $\pm 20^{\circ}$ in any direction.

Numeric data acquisition system (SPIDER 8) is an electronic system for measuring analog numerical data, specialized for numerical acquisition of mechanical quantities (forces, mechanical stress, pressure, acceleration, velocity, displacement, temperature). It also has the possibility to measure the voltage, which allows measurement of any parameter, with the condition of interconnection in the measuring system of another system to convert the voltage signal of that parameter.

Analysis of the dynamic behavior of the mechanical system was performed with the program DinamicaRobot.tst. It was intended: data visualization, time and frequency domain analysis and visualization of graphics and cinematic dynamic of the robotic arm under test conditions. This is a product under TestPoint programming environment used for simulating and analyzing dynamic systems. TestPoint provides the user with a powerful mathematical library for analyzing digital signals in both time and frequency domains and control a wide range of data acquisition systems.

\section{Results and discussion}

The first experiment refers to the ability of spatial movement of the poly-articulated robotic arm as a function of the forces that manifest themselves in the tension cables of the outer mechanism; tension cables were successively connected to the outer mechanism, one by one, the SL2 linear sled through a TF50N force transducer. Thus, it can be determinate the response of the end effector (outer distal vertebra) to a different scaling of the driving force from each cable. Figure 1 shows a cross section through the poly-articulated robotic arm, highlighting the position of the tension cable (wire).

From experimental functional analysis of general assembly, it results that it is necessary to know the spatial coordinates of the following elements:

- linear longitudinal movement of the sled 1, SL1 - XL1 (mm);

- linear longitudinal movement of the sled 2, SL2 - XL2 (mm);

- OX movement direction of the distal vertebra of the robotic arm, XB (mm);

- OY movement direction of the distal vertebra of the robotic arm, YB (mm);

- OZ movement direction of the distal vertebra of the robotic arm, ZB (mm); 


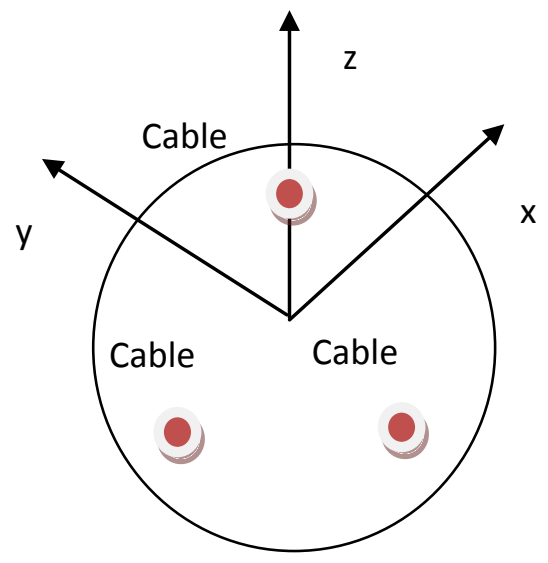

Figure 1: Position of the three tension cables

Was tensioned the cable 1 using a controlled position actuator. Each cable connected rigidly to a single link (distal) and slides along the other vertebrae of the robotic arm. The tension cable 1 was fix to the linear sled SL2. Then, was applied a progressively increasing force to the cable by moving the slide SL2 linear to OX direction. Force is measured with TF50N force transducer. Sampling frequency acquisition system SPIDER 8 was $300 \mathrm{~Hz}$.

Polynomial interpolation was performed on increasing portion of the characteristics experimentally determined between time points $8.305 \mathrm{~s} \div 54.59 \mathrm{~s}$ and on the decreasing portion between time points $90.095 \mathrm{~s} \div 143.15$ s, using polynomials as:

$\mathrm{f}(\mathrm{t})=\mathrm{a}+\mathrm{bt}+\mathrm{ct} \mathrm{t}^{2}+d \mathrm{t}^{3}$

Polynomial interpolation parameters and time points in which interpolation was performed are shown in Table 1.

Following the development of experimental tests on dynamic behavior in time domain of the poly-articulated robotic arm, in accordance with the test program, we obtained some results, tables and graphs on the time variation of the main dynamic parameters. Using graphics facilities of 16 channels, in Table 2 is given a selection of representative values of parameters at different points in time and in Figure 2 is the graphic parameter variation of XL2 F, XB, YB and ZB in the first tension cable.

Amplitude disturbance was considered uniform and the characteristics of the parameter values varied, in terms of size, around the unit. Negative force means that we have traction force in the cable. At the reverse movement of the sled, there is a jump of traction force due to the rotation with a small angle of the cable grip system.

Table 1: Polynomial interpolation constants at the tensioned cable (wire) 1

\begin{tabular}{|c|c|c|c|c|c|c|c|}
\hline \hline Fil_Name & Parameters & T_Inf (s) & T_Sup (s) & a & b & c & d \\
\hline \hline \multirow{5}{*}{ Cable 1 } & XSL2 [mm] & 8.305 & 54.59 & -30.0393 & 0.423906 & 0.002707 & $-4.24 \mathrm{E}-05$ \\
\cline { 2 - 8 } & F [N] & 8.305 & 54.59 & -549.927 & -0.00073 & -0.04937 & 0.00065 \\
\cline { 2 - 8 } & XB [mm] & 8.305 & 54.59 & 23.73675 & -4.22077 & 0.163584 & -0.00087 \\
\cline { 2 - 8 } & YB [mm] & 8.305 & 54.59 & 18.18544 & -2.40741 & 0.065025 & $1.62 \mathrm{E}-05$ \\
\cline { 2 - 8 } & ZB [mm] & 8.305 & 54.59 & 4.998807 & -1.30944 & 0.124863 & -0.00094 \\
\cline { 2 - 8 } & XSL2 [mm] & 90.095 & 143.15 & 251.3527 & -6.01453 & 0.046807 & -0.00013 \\
\cline { 2 - 8 } & F [N] & 90.095 & 143.15 & -565.108 & -0.52137 & 0.011357 & $-4.61 \mathrm{E}-05$ \\
\cline { 2 - 8 } & XB [mm] & 90.095 & 143.15 & 3907.946 & -91.254 & 0.718703 & -0.00189 \\
\cline { 2 - 8 } & YB [mm] & 90.095 & 143.15 & 4593.75 & -110.652 & 0.882505 & -0.00234 \\
\cline { 2 - 8 } & ZB [mm] & 90.095 & 143.15 & 3853.668 & -85.772 & 0.641143 & -0.00161 \\
\hline
\end{tabular}


The second experiment concerns the elasticity of the tension cables. Slowing the speed movement of the robotic arm is because cables (wires) are at the limit of traction performance. Those used are made of a cable that has in his structure steel wire with elastic modulus $\mathrm{E}=2.1 \times 10^{5} \mathrm{MPa}$ and transverse contraction coefficient 0.28 .

Table 2: Values of parameters XL2, F, XB, YB and ZB at the tensioned cable (wire) 1

\begin{tabular}{|c|c|c|c|c|c|c|}
\hline Fil_Name & $\mathrm{T}[\mathrm{s}]$ & XL2[mm] & $\mathrm{F}[\mathrm{N}]$ & $\mathrm{XB}[\mathrm{mm}]$ & $\mathrm{YB}[\mathrm{mm}]$ & $\mathrm{ZB}[\mathrm{mm}]$ \\
\hline \multirow{14}{*}{ Cable 1} & 7.195 & -0.00051 & -0.10877 & $1.60 \mathrm{E}-14$ & 0 & -0.15175 \\
\hline & 14.475 & 3.838295 & -9.75284 & 0.156305 & 0 & 9.433963 \\
\hline & 20.7 & 6.430195 & -15.5393 & 3.891959 & -0.00626 & 22.35988 \\
\hline & 24.9 & 8.760413 & -23.0679 & 12.072 & 0 & 37.00165 \\
\hline & 30.3 & 11.29624 & -26.4278 & 23.31959 & 4.072049 & 49.69783 \\
\hline & 35.15 & 13.72574 & -32.9608 & 43.63389 & 13.85964 & 71.48449 \\
\hline & 40.7 & 16.32784 & -38.3117 & 72.41092 & 33.04657 & 98.05685 \\
\hline & 46.55 & 18.58311 & -42.2938 & 99.65116 & 52.43173 & 120.4943 \\
\hline & 52.2 & 20.88634 & -40.7383 & 133.0438 & 74.29414 & 144.3675 \\
\hline & 62.485 & 23.96506 & -36.134 & 142.3612 & 86.1765 & 152.7164 \\
\hline & 70.815 & 23.96506 & -35.7607 & 142.3665 & 86.1765 & 152.5449 \\
\hline & 79.145 & 23.96506 & -35.3874 & 142.3717 & 86.1765 & 152.5449 \\
\hline & 85.85 & 23.96506 & -35.1385 & 142.377 & 86.1765 & 152.5449 \\
\hline & 89.45 & 22.04548 & -6.14409 & 140.6456 & 81.05057 & 147.4773 \\
\hline
\end{tabular}

Figure 3 represents the behavior of the drive cable at a force of $0.310 \mathrm{kN}$. Increased stiffness of the system has the following effects: providing a more precise control in terms of orientation of the robotic arm, increasing the diameter of the central wire and adoption of a material different from the one used before. Figure 5 represents the critical point where the cable breaks.

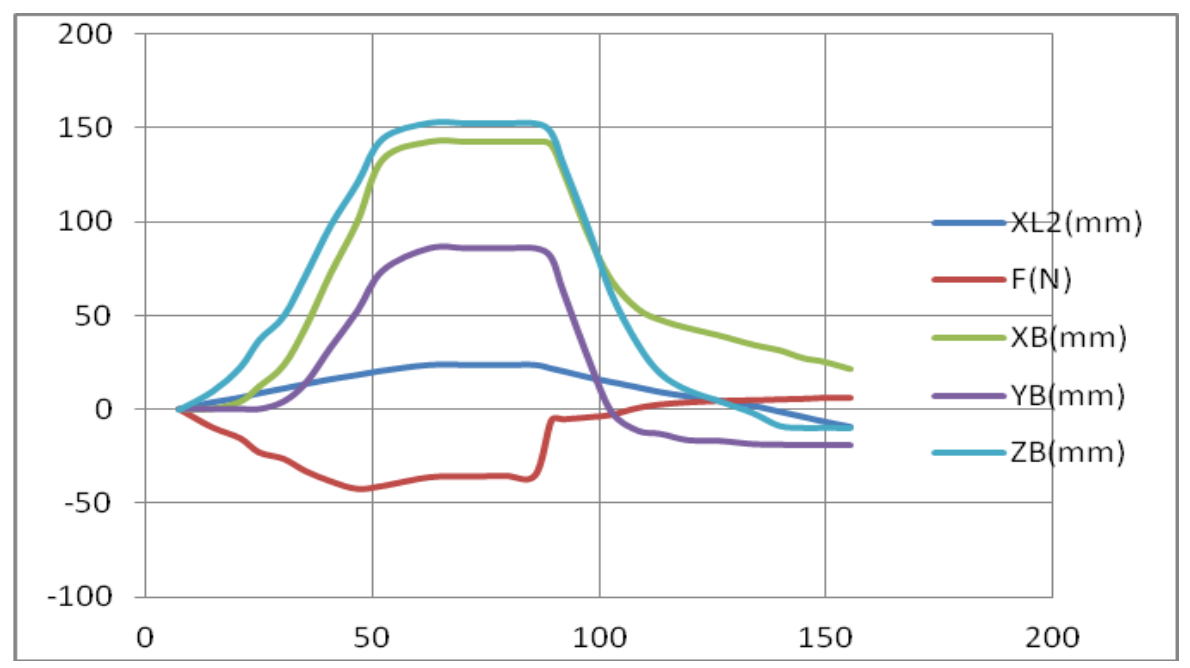

Figure 2: Graphical representation of the variation parameters XL2, F, XB, YB and ZB. 

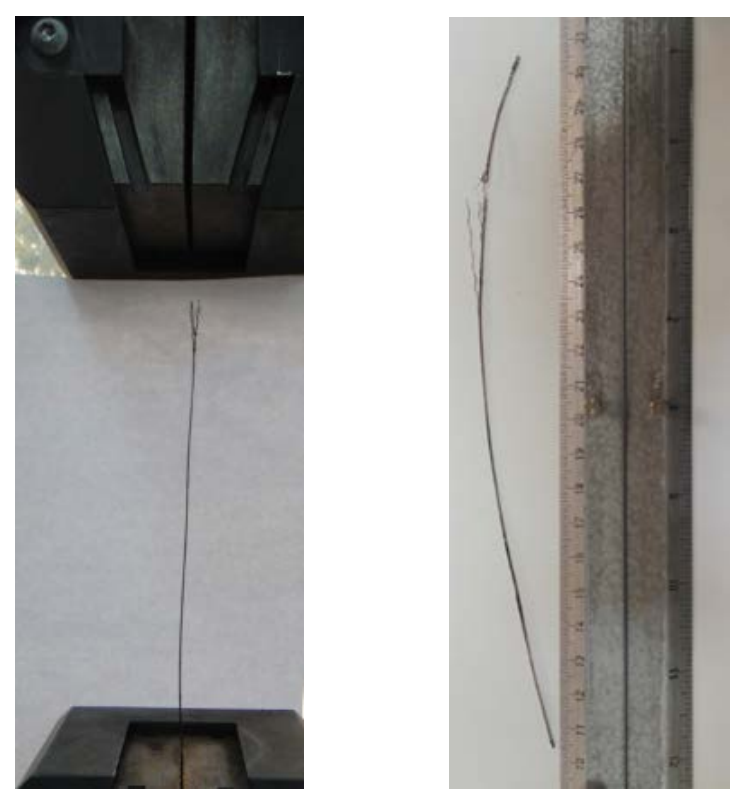

Figure 3: Behavior of the drive cable from a force of $0.310 \mathrm{kN}$

The results test gave the following technical characteristics: mechanical resistance $\mathrm{Rm}=682274$ and elongation $\mathrm{A}=35.6 \%$. Changing the drive cable elasticity is better for the robotic arm rigidity and for a higher precision in the robotic arm actuation.

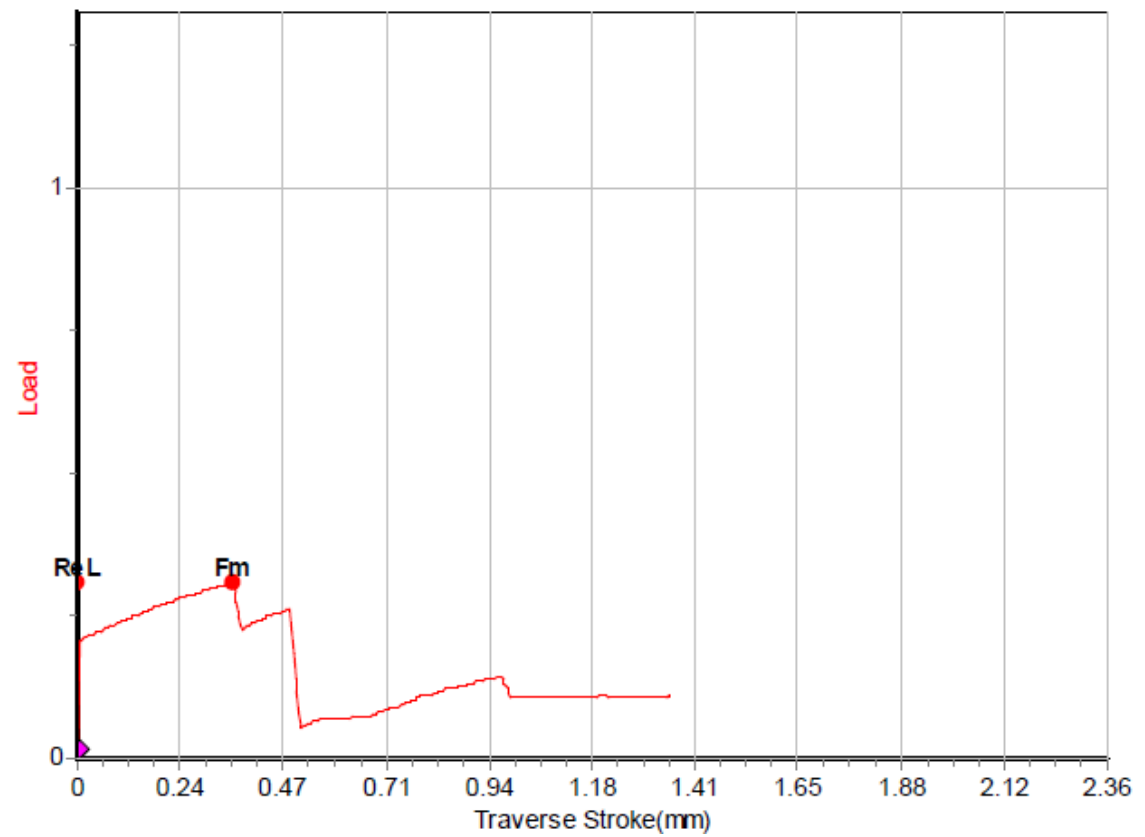

Figure 5: Transverse stroke of the cable depending on load

\section{Conclusions}

To the knowledge of the dynamic behavior of robots, we used a modular test stand which allows the necessary experiments to obtain graphical features of dynamic parameters (force, displacement etc.).

The experimental tests were carried out to verify the mechanical and technological tests and kinematic and dynamic stability of the mechanical system. Tests have validated the constructive solutions chosen, for determining the factors that influence the dynamic behavior of poly-articulated precision robots. 
Mechanical system was analyzed using simulation and analysis tool DimamicaRobot.tst under TestPoint programming environment. TestPoint modules package enables testing of mechanical systems with high complexity, with minimal time allocated for programming and virtual representation of the structural scheme.

In future, there are some research directions regarding robots: reducing and optimization of design components, increase range of motion and development of feedback programs.

\section{Acknowledgment}

This work was supported by the strategic grant POSDRU/159/1.5/S/133255, Project ID 133255 (2014), co-financed by the European Social Fund within the Sectorial Operational Program Human Resources Development 2007 - 2013.

\section{References}

1. Taylor, R., Stoianovici, D., "Medical robotics in computer integrated surgery", IEEE Transaction on Robotics and Automation, vol. 19, pp. 765-781, 2003.

2. Hirose, S., "Biologically inspired robots: snake-like locomotors and manipulators," Applied Mechanics Reviews, Oxfort University Press, vol. 48, pp. 27, 1995.

3. Dario, P., Paggetti, C., Troisfontaine, N., Papa, E., Ciucci, T., Carrozza, M., C., Marccaci, M., “A miniature steerable end-effector for application in an integrated system for computer-assisted arthroscopy”, IEEE International Conference on Robotics and Automation,, pg. 1573-1579, 1997.

4. Reynaerts, D., Peirs, J., Van Brussel, H., "Shape memory microactuation for a gastro-intestinal intervention system," Sensors and Actuators, A: Physical, vol. 77, pp. 157, 1999.

5 Guthart, G., Salisbury, K., „The intuitive tele-surgery; overview and application” IEEE International Conference on Robotics and Automation, pp. 618- 621, 2000.

6. Dumitru V., PHD thesis: Poli-articulated mechanical system used in medical domain, Craiova, 2011.

7 Dumitru V., Modular System for Testing the Performance of Poly-articulate Robotic Structure, International Coferince on Mechanical Engineering - ICOME 2013, 12th - 14th September 2013, Mataram, Lombok, Indonezia, Applied Mechanics and Materials;ISBN:978-3-03785-9902;ISSN: 1660-9336, pp:414-419. 